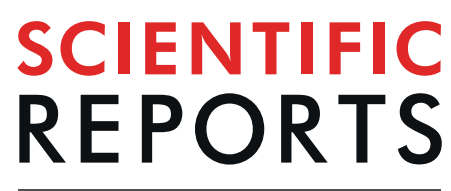

natureresearch

\title{
OPEN Muscle transcriptome analysis reveals genes and metabolic pathways related to mineral concentration in Bos indicus
}

Received: 31 January 2019

Accepted: 2 August 2019

Published online: 03 September 2019

\author{
Juliana Afonso $\mathbb{1 D}^{1}$, Luiz Lehmann Coutinho $\mathbb{1}^{2}$, Polyana Cristine Tizioto ${ }^{8}$, \\ Wellison Jarles da Silva Diniz $\mathbb{1}^{1}{ }^{1}$, Andressa Oliveira de Lima ${ }^{1}$, Marina Ibelli Pereira Rocha ${ }^{1}$, \\ Carlos Eduardo Buss ${ }^{1}$, Bruno Gabriel Nascimento Andrade ${ }^{3}$, Otávio Piaya ${ }^{1}$, \\ Juliana Virginio da Silva ${ }^{4}$, Laura Albuquerque Lins ${ }^{5}$, Caio Fernando Gromboni ${ }^{6}$, \\ Ana Rita Araújo Nogueira ${ }^{3}$, Marina Rufino Salinas Fortes ${ }^{7}$, Gerson Barreto Mourao ${ }^{2} \&$ \\ Luciana Correia de Almeida Regitano $\mathbb{1}^{3}$
}

Mineral content affects the biological processes underlying beef quality. Muscle mineral concentration depends not only on intake-outtake balance and muscle type, but also on age, environment, breed, and genetic factors. To unveil the genetic factors involved in muscle mineral concentration, we applied a pairwise differential gene expression analysis in groups of Nelore steers genetically divergent for nine different mineral concentrations. Here, based on significant expression differences between contrasting groups, we presented candidate genes for the genetic regulation of mineral concentration in muscle. Functional enrichment and protein-protein interaction network analyses were carried out to search for gene regulatory processes concerning each mineral. The core genetic regulation for all minerals studied, except $\mathrm{Zn}$, seems to rest on interactions between components of the extracellular matrix. Regulation of adipogenesis-related pathways was also significant in our results. Antagonistic patterns of gene expression for fatty acid metabolism-related genes may explain the $\mathrm{Cu}$ and $\mathrm{Zn}$ antagonistic effect on fatty acid accumulation. Our results shed light on the role of these minerals on cell function.

The role of minerals in meat quality traits is perceived in the nutritional value of beef. For example, high iron content is a major player in the claims regarding nutritional value ${ }^{1}$. The second meat quality trait affected by minerals is beef tenderness since calcium related-proteases take part in the post-mortem degradation of myofibrillar proteins ${ }^{2}$. As minerals are essential for a variety of biological processes such as metabolism, homeostasis maintenance, growth, influencing cellular structural components, enzyme cofactors, regulation of cell replication, and differentiation ${ }^{3}$, they may affect other economically important traits in livestock production.

Mineral concentration in mammalian muscles depends on animal intake-outtake imbalance ${ }^{4}$, muscle type $e^{5}$, age, environment ${ }^{6}$, breed ${ }^{7}$, and other genetic factors ${ }^{8}$. Minerals can only perform their biological function in muscle cells if they are available in the right amount ${ }^{3}$. Their concentration is under strict control for homeostasis maintenance. The genetic control of mineral homeostasis is not fully understood, although there is evidence regarding specific genes and certain gene functions linked to mineral concentration in many species. Calcium concentration in humans depends upon a complex network of hormones ${ }^{9}$. Both serum and urinary calcium were deemed continuous heritable traits, in a study with twins ${ }^{9}$. Genes related to sodium and potassium homeostasis

\footnotetext{
${ }^{1}$ Department of Evolutionary Genetics and Molecular Biology, Federal University of São Carlos, São Carlos, Brazil. ${ }^{2}$ Department of Animal Science, University of São Paulo/ESALQ, Piracicaba, Brazil. ${ }^{3}$ Embrapa Pecuária Sudeste, São Carlos, Brazil. ${ }^{4}$ Physics Institute of São Carlos, University of São Paulo, São Carlos, Brazil. ${ }^{5}$ Animal Science department, Laboratory of Molecular Genetics. São Paulo State University, Jaboticabal, Brazil. ${ }^{6}$ Bahia Federal Institute of Education, Science and Technology, Ilhéus, Brazil. ${ }^{7}$ School of Chemistry and Molecular Biosciences, Faculty of Sciences, The University of Queensland, Brisbane, Australia. ${ }^{8} \mathrm{NGS}$ Soluções Genômicas, Rua Ajudante Albano 847, Piracicaba, SP, Brazil. Correspondence and requests for materials should be addressed to L.C.d.A.R. (email: luciana.regitano@embrapa.br)
} 


\begin{tabular}{|l|l|l|c|c|l|l|l|}
\hline Group & GEBV & St. Error $^{\mathbf{a}}$ & Concentration $^{\mathbf{b}}$ & St. Error $^{\mathbf{c}}$ & Read aligned pairs & Alignment (\%) $^{\text {T-test p-value }}$ \\
\hline Low-Ca & -0.1122 & 0.007 & 85.75 & 9.1261 & $10,103,844$ & 93.98 & \\
\hline High-Ca & 0.1366 & 0.0247 & 346.71 & 31.3709 & $16,664,657$ & 91.48 & $8.55 \mathrm{E}-05$ \\
\hline Low-Cu & -0.0607 & 0.0015 & 1.13 & 0.0396 & $10,280,771$ & 92.9 & \\
\hline High-Cu & 0.1228 & 0.0378 & 4.46 & 1.6121 & $12,204,273$ & 92.15 & $4.70 \mathrm{E}-03$ \\
\hline Low-K & -0.0447 & 0.0035 & 976.05 & 26.2935 & $14,682,381$ & 91.22 & \\
\hline High-K & 0.0872 & 0.0028 & 2152.36 & 65.5082 & $13,526,576$ & 91.63 & $1.30 \mathrm{E}-10$ \\
\hline Low-Mg & -0.0435 & 0.0026 & 668.6 & 14.788 & $14,682,381$ & 91.22 & \\
\hline High-Mg & 0.0773 & 0.0038 & 1401.05 & 32.1704 & $15,112,850$ & 91.67 & $1.05 \mathrm{E}-09$ \\
\hline Low-Na & -0.0478 & 0.0035 & 1544.6 & 57.8293 & $13,098,861$ & 91.57 & \\
\hline High-Na & 0.097 & 0.0047 & 3807.57 & 136.1052 & $15,112,850$ & 91.67 & $1.05 \mathrm{E}-09$ \\
\hline Low-P & -0.0459 & 0.002 & 6354.98 & 232.1688 & $13,827,066$ & 91.68 & \\
\hline High-P & 0.0843 & 0.0031 & 14128.47 & 523.2822 & $13,526,576$ & 91.63 & $1.30 \mathrm{E}-10$ \\
\hline Low-S & -0.061 & 0.0017 & 4650.32 & 260.7678 & $11,665,773$ & 92.07 & \\
\hline High-S & 0.0832 & 0.0041 & 11783.28 & 588.4472 & $14,037,706$ & 91.23 & $1.63 \mathrm{E}-08$ \\
\hline Low-Se & -0.1703 & 0.0087 & 0.0765 & 0.0052 & $12,902,391$ & 91.97 & \\
\hline High-Se & 0.1143 & 0.0066 & 0.32 & 0.0209 & $10,678,101$ & 91.05 & $5.02 \mathrm{E}-10$ \\
\hline Low-Zn & -0.071 & 0.0058 & 58.59 & 2.6137 & $13,370,593$ & 91.98 & \\
\hline High-Zn & 0.1115 & 0.0061 & 183.17 & 9.4696 & $14,531,511$ & 91.63 & $1.04 \mathrm{E}-09$ \\
\hline
\end{tabular}

Table 1. Statistics of the genetic estimated breeding values and RNA-Seq for each extreme group. All values

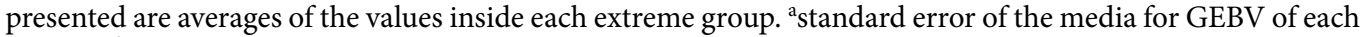
mineral, baverage mineral concentration in $\mathrm{mg} / \mathrm{Kg}$ for each extreme group, ${ }^{\mathrm{c}}$ standard error of the media for the mineral concentration of each mineral, ${ }^{\mathrm{d}} \mathrm{p}$-value of the test of significance ( $\mathrm{t}$-test) between the extreme group samples' GEBVs for each mineral.

in humans were reviewed elsewere ${ }^{10}$. Magnesium absorption in bovine is breed dependent ${ }^{11}$. Zinc homeostasis is poorly understood, but in C. elegans there is a conserved motif called low zinc activation element in promoters that seems to be involved in the process ${ }^{12}$. Genes associated to copper transport in higher eukaryotes (hCTR1/2, Atox1 and Atp7A/B) were detected in a yeast functional screen that aimed to find genes linked to copper-dependent respiratory growth, which could be candidate markers to human mitochondrial diseases ${ }^{13}$. Genes related to iron concentration participate in lipid metabolism in Nelore cattle ${ }^{14}$. Still, information about genes associated with mineral composition in beef is scarce.

Scientific evidence about genetic mechanisms associated with bovine mineral deposition regulation in muscle comes from a limited number of studies. Quantitative trait loci (QTLs) related to mineral concentration were described in Jersey x Limousin crosses ${ }^{4}$, Nelore ${ }^{15}$, Holstein, and Jersey ${ }^{16}$ cattle. These studies reported some overlapping QTLs and enriched functional processes among different minerals, indicating shared genetic regulation.

Once minerals participate in a variety of biochemical processes that might affect production traits, understanding the genetic and physiological processes underlying muscle mineral concentration might provide the basis for modulating these processes to the benefit of cattle production. Selective breeding could incorporate gene polymorphisms that influence mineral composition to improve the nutritional value of beef ${ }^{17}$. Understanding the genetics and gene regulation associated with muscle minerals in cattle may also provide some evidence for how conserved these are across species. Given biochemical similarities across mammals, it is possible that increased knowledge from cattle studies might be generalized to humans.

Herein, to characterize the biological pathways involved in muscle mineral deposition, we described a differential expression RNA-seq analysis from Longissimus thoracis muscle of contrasting mineral content Nelore steers, pinpointing genes, processes, and pathways related to mineral homeostasis. The minerals analyzed were Calcium (Ca), Copper $(\mathrm{Cu})$, Potassium (K), Magnesium (Mg), Sodium (Na), Phosphorus (P), Sulfur (S), Selenium (Se), and Zinc (Zn).

\section{Results}

Animals and RNA-Seq analysis. Each contrasting group for a specific mineral was called Low (L) or High $(\mathrm{H})$ and differentiated by the corresponding mineral symbol. Due to the overlapping of samples among groups, 44 samples comprised our 18 groups. The average $\mathrm{GEBV}^{15}$ and mineral concentration for contrasting groups confirmed they were significantly different and comparable, as shown in Table 1 . The average number of read pairs aligned was $13,333,842$, and the average percentage of reads aligned to the reference Bovine Genome (UMD 3.1 ) was $91.82 \%$. Our sequencing allowed the identification of a significant number of transcripts. The transcripts discovery saturation curves (discovered transcripts versus reads sequenced) from the samples assessed here are shown in Supplementary Fig. S1.

We identified 29,312 transcripts but tested only 15,012 for differential expression due to their expression levels, since Cuffdiff v2.2.1 ${ }^{18}$ parameters were set to do not take into account genes with less than ten reads aligned, in both differential expression analysis and multiple test correction.

Differentially expressed genes (DEGs). We identified 327 annotated DEGs considering all minerals. The number and annotation status of the DEGs were variable among the evaluated groups (Table 2). All DEGs and 


\begin{tabular}{|l|l|l|l|l|l|l|l|l|l|}
\hline Mineral & Ca & Cu & P & Mg & K & Se & Na & Zn & S \\
\hline Annotated genes $^{\mathrm{a}}$ & 170 & 125 & 43 & 53 & 51 & 25 & 55 & 27 & 15 \\
\hline Predicted proteins $^{\mathrm{b}}$ & 24 & 7 & 6 & 5 & 5 & 6 & 5 & 4 & 3 \\
\hline Non-annotated genes $^{\mathrm{c}}$ & 35 & 23 & 17 & 22 & 23 & 6 & 13 & 4 & 4 \\
\hline Upregulated $^{\mathrm{d}}$ & 34 & 13 & 8 & 10 & 9 & 9 & 13 & 15 & 6 \\
\hline Downregulated $^{\mathrm{e}}$ & 160 & 119 & 41 & 48 & 47 & 22 & 47 & 16 & 12 \\
\hline Total & 229 & 155 & 66 & 80 & 79 & 37 & 73 & 35 & 22 \\
\hline
\end{tabular}

Table 2. Number and Annotation status of DEGs per mineral. ${ }^{a}$ Genes with known annotation based on the

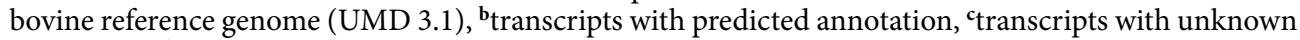
annotation, ${ }^{d}$ annotated genes and predicted proteins more expressed in the high groups, ${ }^{\text {e }}$ annotated genes and predicted proteins more expressed in the low groups.

their fold change (FC) values between contrasting groups for each mineral are in Supplementary Table S1. There were no common DEGs to all minerals. However, 27genes were common to at least five minerals. From these, we can highlight COL11A1, COMP and TNMD genes, common to eight minerals (all, except $\mathrm{Zn}$ ). The minerals with more DEGs overlapping were $\mathrm{Mg}, \mathrm{Na}, \mathrm{K}$, and $\mathrm{P}$, with 25 . In all expression comparisons between contrasting groups, upregulation means higher expression in H-groups than in L-groups. Conversely, downregulation means lower expression in H-groups than in L-groups. Unlike $\mathrm{Zn}$, which had 50\% of the DEGs downregulated, the remaining minerals presented at least $66 \%$ of the DEGs as downregulated.

DEGs with the highest estimated FC ( $>1.9$ ) between each contrasting mineral group were $M T 2 A$ for $\mathrm{K}, \mathrm{Mg}$, $\mathrm{Na}$, and P; RN5-8S1 for Se and S; HSPA6 for $\mathrm{Cu}, \mathrm{Zn}, \mathrm{P}$ and Se; PMP2 for $\mathrm{Cu}$, and GBP4 for Ca. DEGs with lowest FC ( < 1.9) between groups were TNMD, COMP, and COL11A1 for eight minerals (except for Zn); FBLN7 in seven of them (except for S and Zn), and CILP2 in six (except for S, Zn, and Na). Among DEGs with the lowest FC (downregulated in the $\mathrm{H}$-group) for at least two minerals, we found $P E R P$ for $\mathrm{Cu}, \mathrm{K}, \mathrm{Mg}, \mathrm{P}$, and $\mathrm{Se}$; TNC and THBS4 for $\mathrm{Cu}, \mathrm{K}, \mathrm{Mg}$, and $\mathrm{P}$; COL22A1 for $\mathrm{Cu}, \mathrm{Na}, \mathrm{P}$, and Se; $A D A M 12$ for $\mathrm{Cu}$ and Se; $A C T C 1$ for $K$ and $P$; CRABP2 and CRTAC1 for $\mathrm{K}, \mathrm{Mg}$, and $\mathrm{P}$; KCNK2, MKX and MXRA5 for $\mathrm{Ca}$ and $\mathrm{Cu}$.

Regarding individual minerals, we found TF, HOXA9, MIR196B, and SYT4 genes as top downregulated in H-Ca group; ELOVL6, PTGIR, COL12A1, GAS2, POSTN, WISP1, MLLT11, and THRSP for Cu; PIIS for Na; $M Y L K 3$ for S; and RN5-8S1 for Zn. From Se and S analyses, RN5851 gene was upregulated in higher mineral concentration group.

Functional enrichment analysis. We performed a functional annotation analysis applying the Trinotate pipeline (http://trinotate.sourceforge.net/) to identify possible biological functions of non-annotated DEGs. We retrieved possible functions for 31 transcripts. From these, 18 presented functions related to LINE-1 retrotransposable elements, retrovirus-related Pol polyprotein, and immune response related functions. We also recovered the function "similar to the protein SAMHD1", a restriction nuclease that suppresses LINE-1 retrotransposition activity $^{19}$ (Supplementary Table S2). Among the non-annotated transcripts from Cu DEGs, one is highly similar to a myoregulin (GO: 0016021), with high homology to a human MRLN gene (91.30\% of similarity). Another one was annotated as the Sentrin-specific protease 3, having homology with a mouse SENP3 gene $(92.86 \%$ of similarity).

We clustered annotated functions obtained with DAVID software ${ }^{20}$ for each predicted protein whose coding gene was a DEG for each mineral. The summarized significant analysis is presented in Table 3 . We did not obtain substantial annotated function clusters for $\mathrm{Zn}$ and $\mathrm{S}$. Common functions in at least four minerals were related to the extracellular matrix (enriched in seven minerals), extracellular matrix-receptor interaction (ECM-receptor interaction), collagen and secretion, the latest three enriched in six minerals. Also, for five minerals we identified disulfide bond, epidermal growth factor-like domain, focal adhesion and, for four minerals, protein digestion and absorption.

Relationship among minerals. The GEBVs for most mineral concentrations showed significant Pearson correlations in our population, ranging from -0.2 to 0.97 (Supplementary Table S3). Also, high significant correlations were observed between each GEBV and their correspondent raw mineral concentrations, varying from 0.77 to 0.86 (Supplementary Table S4). Results of t-tests to verify if the mean GEBVs of the samples used to represent the contrasting groups for one mineral would also be statistically different for any other mineral are shown in Supplementary Table S5.

Protein-protein interaction and pathways among DEGs. To identify biological processes involving the DEGs, we performed a protein-protein interaction (PPI) network analysis among DEGs for each mineral using STRING v.1.2.2 software ${ }^{18}$, which retrieves pathways from KEGG database ${ }^{21}$. DEGs for each mineral partaking in known PPI, and its significant pathways, are shown in Fig. 1. Sulfur did not present a significant pathway.

All DEGs presented in the same pathway for a given mineral had the same direction of expression, i.e., they were either all upregulated or all downregulated (Supplementary Table S1). DEGs presented in each pathway across mineral analyses can be seen in Fig. 2. Significant pathways for all minerals are shown in Table 4. ECM-receptor interaction pathway was common to seven minerals (except $\mathrm{Zn}$ and S), protein digestion and 


\begin{tabular}{|c|c|c|c|c|c|c|}
\hline $\mathrm{Ca}$ & $\mathrm{Cu}$ & $\mathbf{P}$ & $\mathrm{Mg}$ & $\mathbf{K}$ & Se & $\mathrm{Na}$ \\
\hline Cell-cell interaction & Calcium ion binding & Cell adhesion & Carboxypeptidase & Collagen & Extracellular matrix & Carboxypeptidase \\
\hline Collagen & Carboxypeptidase & Collagen & Collagen & Disulfide bond & & Cell adhesion \\
\hline $\begin{array}{l}\text { ECM-receptor } \\
\text { interaction }\end{array}$ & Cell adhesion & Disulfide bond & Disulfide bond & $\begin{array}{l}\text { ECM-receptor } \\
\text { interaction }\end{array}$ & & Collagen \\
\hline Extracellular matrix & Cell-cell interaction & $\begin{array}{l}\text { ECM-receptor } \\
\text { interaction }\end{array}$ & $\begin{array}{l}\text { ECM-receptor } \\
\text { interaction }\end{array}$ & $\begin{array}{l}\text { Epidermal growth } \\
\text { factor-like domain }\end{array}$ & & Disulfide bond \\
\hline $\begin{array}{l}\text { Protein digestion and } \\
\text { absorption }\end{array}$ & Collagen & $\begin{array}{l}\text { Epidermal growth } \\
\text { factor-like domain }\end{array}$ & $\begin{array}{l}\text { Epidermal growth } \\
\text { factor-like domain }\end{array}$ & Extracellular matrix & & $\begin{array}{l}\text { ECM-receptor } \\
\text { interaction }\end{array}$ \\
\hline Proteoglycans & Disulfide bond & Extracellular matrix & Extracellular matrix & Focal adhesion & & $\begin{array}{l}\text { Epidermal growth } \\
\text { factor-like domain }\end{array}$ \\
\hline Secretion & $\begin{array}{l}\text { ECM-receptor } \\
\text { interaction }\end{array}$ & Focal adhesion & Focal adhesion & Glycoprotein & & Extracellular matrix \\
\hline \multirow[t]{8}{*}{ Signaling } & $\begin{array}{l}\text { Epidermal growth } \\
\text { factor-like domain }\end{array}$ & $\begin{array}{l}\text { PI3K-Akt signaling } \\
\text { pathway }\end{array}$ & Glycoprotein & $\begin{array}{l}\text { Immunoglobulin-like } \\
\text { domain }\end{array}$ & & Focal adhesion \\
\hline & Extracellular matrix & $\begin{array}{l}\text { Protein digestion and } \\
\text { absorption }\end{array}$ & Secretion & Secretion & & Glycoprotein \\
\hline & Fatty acid metabolism & Secretion & & & & Leucine-rich repeat \\
\hline & Focal adhesion & & & & & $\begin{array}{l}\text { Protein digestion and } \\
\text { absorption }\end{array}$ \\
\hline & $\begin{array}{l}\text { PI3K-Akt signaling } \\
\text { pathway }\end{array}$ & & & & & Secretion \\
\hline & $\begin{array}{l}\text { Protein digestion and } \\
\text { absorption }\end{array}$ & & & & & \\
\hline & Secretion & & & & & \\
\hline & Signaling & & & & & \\
\hline
\end{tabular}

Table 3. DEGs summarized significative annotated function clusters. The results were obtained using DAVID software. There are no significative results for $\mathrm{Zn}$ e S. Results are displayed for each mineral and in alphabetic order.

absorption pathway was common to six (except Zn, S, and K), and focal adhesion pathway and PI3K-Akt signaling pathway to five (except $\mathrm{Ca}, \mathrm{Se}, \mathrm{Zn}$, and S). All DEGs presented in these pathways were downregulated.

Fatty acid metabolism pathway was common to $\mathrm{Zn}$ and $\mathrm{Cu}$. This was the only pathway where DEGs had different regulation between both minerals. Of all DEGs in this pathway, three were common for both minerals (ELOVL6, FASN, and SCD) and two were exclusive to Cu concentration analysis (ELOVL5 and ACACA) (Fig. 2). From all minerals, $\mathrm{Cu}$ retrieved more enriched pathways $(\mathrm{N}=10)$, whereas prion disease and phagosome pathways were identified only in $\mathrm{Ca}$ and $\mathrm{K}$ analyses, respectively.

After filtering out DEGs that did not interact in our PPI network, 96 remained for $\mathrm{Ca}, 64$ for $\mathrm{Cu}, 17$ for $\mathrm{K}, 18$ for $\mathrm{Mg}, 19$ for $\mathrm{Na}, 20$ for P, two for S, 11 for Se, and 10 for $\mathrm{Zn}$. In total, Ca and Cu had more than 50\% of their DEGs taking part in an interaction (56.47\% and 51.2\%, respectively), $\mathrm{P}$ and Se had around $45 \%$ (46.5\% and $44 \%$, respectively), K, $\mathrm{Na}, \mathrm{Mg}$, and $\mathrm{Zn}$ had about $35 \%(33.3 \%, 34.5 \%, 33.96 \%$, and $37 \%$,) and $\mathrm{S}$ had the lowest rate of DEGs in interactions, $13.3 \%$. From DEGs' products that did not take part in protein-protein interactions, only five were part of a pathway: COL11A2 for $\mathrm{K}, \mathrm{Ca}, \mathrm{Na}, \mathrm{Mg}$ and $\mathrm{Cu}$; COL11A1 for P; CILP2 for K; CD44 for Na; and PTGIR for $\mathrm{Cu}$.

\section{Discussion}

Heritability, GEBVs, and correlations for mineral concentration. Muscle mineral concentrations are moderately heritable traits. Estimates of heritability from our Nelore population ranged from 0.29 to $0.33^{15}$. Understanding the genetic component related to muscle mineral concentration might be useful to better comprehend mineral metabolism and metabolic diseases.

As expected from the correlations among GEBVs for the minerals and from the biological interconnection among them, most contrasting groups for each mineral also differed concerning other mineral's GEBVs, except for $\mathrm{Cu}$ and $\mathrm{Se}$, even though they did not meet the criteria of representing both $5 \%$ extremes from the normal distribution. The most extreme example comes from the minerals $\mathrm{Mg}, \mathrm{Na}, \mathrm{K}$, and $\mathrm{P}$, presenting a correlation higher than 0.88 among their GEBVs. As a consequence, the same samples comprised the low-Mg and low-K, the high-Mg and high-Na and in the high-K and high-P groups. As the complementary contrasting group for each mineral had at least one different sample, the DEGs, functions, and pathways are not entirely the same among these minerals. Given this fact, one should consider that some correlated response regarding other than the mineral in discussion could exist within our results.

$\mathrm{Mg}$ and $\mathrm{K}$, which in our analysis showed a correlation of 0.97 and 46 DEGs in common, presented the same QTLs in previous experiments with this population, thus reinforcing the common genetic control for these minerals. However, Mg and P showed the same pairwise correlation and presented 38 common DEGs, but did not showed QTLs in common ${ }^{15}$. Similarly, despite the correlations, there were no common QTLs among $\mathrm{Mg}, \mathrm{K}, \mathrm{Na}$, and $\mathrm{P}^{15}$. Thus, although not fully explained by pairwise correlation, to some extent, the common DEGs, functions, and pathways among these four minerals can result from the high correlation and sample overlapping among them. 


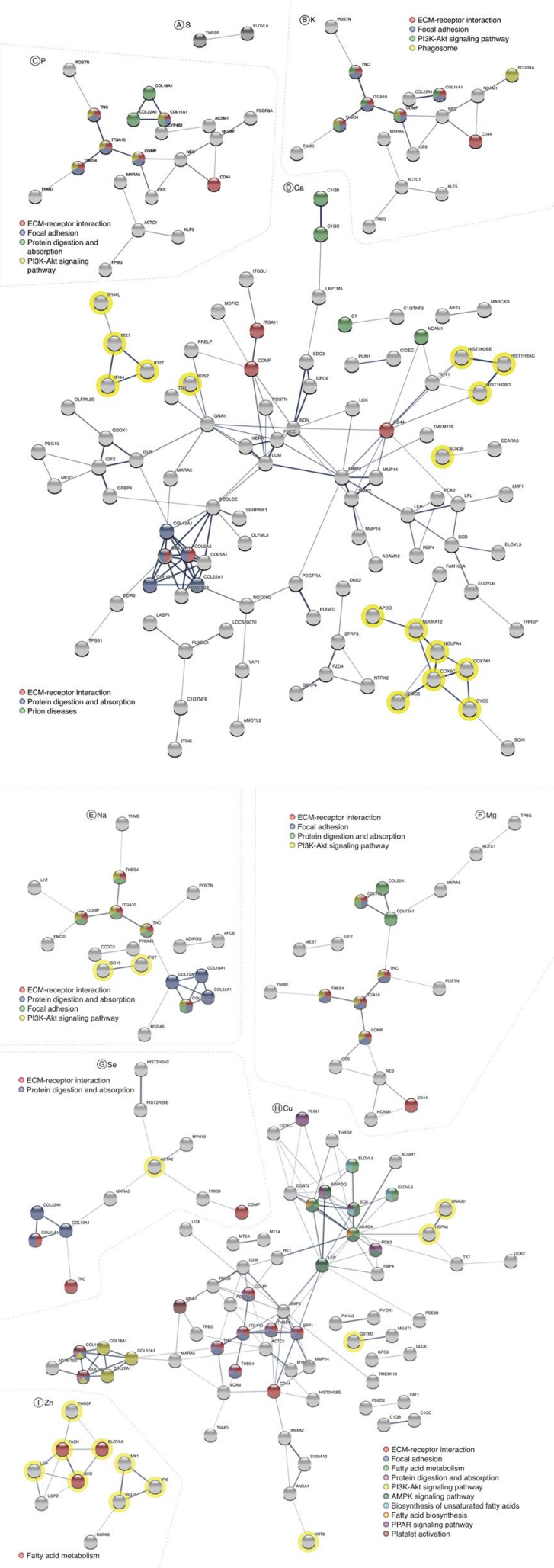

Figure 1. DEGs' products protein-protein interaction network for each mineral. Proteins not partaking in an interaction are not shown. The line thickness between two proteins indicates the strength of data support. The colors inside the circles represent DEGs participating in the same pathway. The yellow halos represent DEGs upregulated in the $\mathrm{H}$ groups in relation to the L groups. The DEGs without a yellow halo were downregulated in the $\mathrm{H}$ groups in relation to L groups. (A) S, (B) K, (C) P, (D) Ca, (E) Na, (F) Mg, (G) Se, (H) Cu, and (I) Zn. 
Pathways

ECM-receptor interaction

Protein digestion and absorption

Prion disease

Focal adhesion

Fatty acid metabolism

PI3K-Akt signaling

AMPK signaling

Biosynthesis of unsaturated fatty acids

Fatty acids biosynthesis

PPAR signaling

Platelet activation

Phagosome
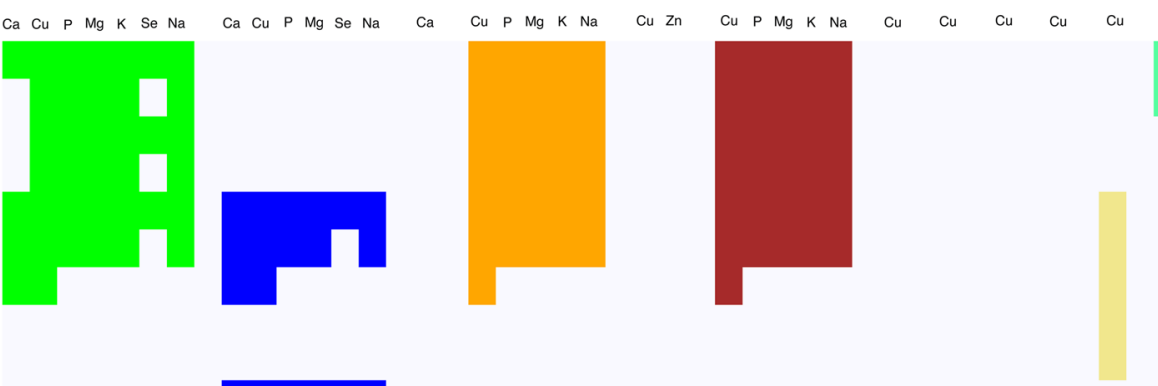

co

THBS

TNG

ITGA10

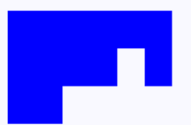

COLI1A1

COL11A2

COL5A2

PTGIR

GNAI1

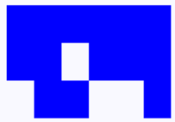

COL22A1

COL12A1

COL18A

c1ac

C1QB

C7

NCAM1

CD44

ITGA11

COL13A

LEP
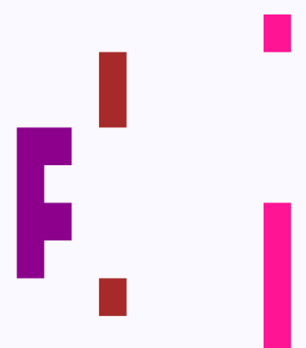

THBS1

SPP1

ELOVL6

ELOVL5

FASM

ACACA

PCK2

ADIPOQ

PLIN1

SCD

Figure 2. DEGs partaking in significant pathways. Rows: DEGs partaking in the significant pathways, columns: minerals presenting the significant pathways, colors: different significant pathways.

\begin{tabular}{|l|l|l|l|l|l|l|l|l|}
\hline KEGG Pathways & $\mathbf{C a}$ & $\mathbf{C u}$ & $\mathbf{P}$ & $\mathbf{M g}$ & $\mathbf{K}$ & $\mathrm{Se}$ & $\mathbf{N a}$ & $\mathbf{Z n}$ \\
\hline ECM-receptor interaction & 0,012 & $2.85 \mathrm{e}-08$ & $1.45 \mathrm{e}-07$ & $5.65 \mathrm{e}-07$ & $4.27 \mathrm{e}-07$ & 0.0229 & $9.57 \mathrm{e}-07$ & - \\
\hline Protein digestion and absorption & 0,012 & 0.0009 & 0.0028 & 0.0060 & - & 0.0229 & 0.0005 & - \\
\hline Focal adhesion & - & 0.0006 & 0.0006 & 0.0019 & 0.0015 & - & 0.002 & - \\
\hline PI3K-Akt signaling pathway & - & 0.0024 & 0.0053 & 0.0153 & 0.0165 & - & 0.023 \\
\hline Fatty acid metabolism & - & 0.0009 & - & - & - & - & - \\
\hline AMPK signaling pathway & - & 0.0056 & - & - & - & - & - & 0.0104 \\
\hline $\begin{array}{l}\text { Biosynthesis of unsaturated fatty } \\
\text { acids }\end{array}$ & - & 0.0121 & - & - & - & - & - \\
\hline Fatty acid biosynthesis & - & 0.0206 & - & - & - & - & - \\
\hline PPAR signaling pathway & - & 0.0283 & - & - & - & - & - \\
\hline Platelet activation & - & 0.0311 & - & - & - & - & - \\
\hline Phagosome & - & - & - & - & 0.0421 & - & - \\
\hline Prion diseases & 0,014 & - & - & - & - & - & - \\
\hline
\end{tabular}

Table 4. DEGs significative KEGG Pathways enriched for each mineral. Sulfur do not present a significative KEGG pathway. P-values displayed for each pathway. 
Detected functions and previous works. All the enriched gene functions convey to one or more enriched pathways. The previous detection of similar functional gene clusters in a GWAS for 14 minerals ${ }^{15}$, whose dataset included our samples, indicates conserved mechanisms affecting mineral concentration. The involvement of common DEGs in shared pathways among minerals reinforces that various genes affect these phenotypes.

Differential expression and QTL analyses can produce similar functional annotation results, but different gene lists due to differences in the methodologies ${ }^{22}$. Our DEGs were not harbored in/or near QTL regions already reported ${ }^{15}$. However, the functional analyses of DEGs and QTLs pointed to similar gene functions. Due to this fact, we will focus our discussion on the genetic similarities among different mineral analyses.

DEGs with opposite FC among minerals. $\quad M T 2 A$ and HSPA6 were the DEGs with the expression pattern presenting the highest FC contrariety and discrepancy among minerals. Both genes have a known relationship with heavy metals. The MT2A gene encodes a metallothionein protein that binds divalent heavy metals and participates in metal control and $\mathrm{Zn}$ homeostasis in the cell, affecting apoptotic and autophagy pathways ${ }^{23}$. MT2A was a DEG for almost all minerals in this study, except $\mathrm{Ca}$ and Se. It is downregulated in the $\mathrm{H}$-Cu group and upregulated in the $\mathrm{H}$-groups of $\mathrm{Mg}, \mathrm{P}, \mathrm{Zn}, \mathrm{Na}, \mathrm{S}$, and $\mathrm{K}$. From these, only $\mathrm{Cu}$ and $\mathrm{Zn}$ are divalent heavy metals. Different polymorphisms in MT2A or its promoter disturb $\mathrm{Zn}$ and Cd concentrations in human blood of healthy patients ${ }^{24}$ and carotid artery stenosis patients ${ }^{25}$. Our results suggest that, apart from the already described in the literature, this gene could also be related to the concentration of other non-heavy metal minerals like $\mathrm{Mg}, \mathrm{K}, \mathrm{Na}$, $\mathrm{P}$, and $\mathrm{S}$.

The HSPA6 gene was upregulated in the $\mathrm{H}-\mathrm{Cu}$ and $\mathrm{H}-\mathrm{S}$ groups, while the opposite occurs for $\mathrm{Zn}$ and $\mathrm{P}$. This gene product responds to stress, and its expression increases with the increase of heavy metal, like $\mathrm{Cu}$, concentration $^{26}$. This protein takes part in the fatty acid metabolism pathway (FAM), where $\mathrm{Zn}$ and $\mathrm{Cu}$ are essential. The relationship between HSPA6, $\mathrm{P}$, and $\mathrm{S}$ is still unknown.

Extracellular matrix interactions. Among the downregulated DEGs with the lowest FC accross minerals, lower than -1.9, we found COMP, COL11A1, TNC, THBS4, and COL22A. They were involved in common pathways for at least six minerals, which may indicate a potential common genetic regulation of mineral concentration or a possible role of mineral concentration in the control of these genes expression. They genes act in pathways such as ECM-receptor interaction, focal adhesion, PI3K-Akt signaling pathway, and protein digestion and absorption. The first three pathways are interconnected (http://www.genome.jp/kegg/pathway/hsa/hsa04510.html).

The DEGs COMP and COL11A1, common to eight minerals, are part of the ECM-receptor pathway. They encode cell membrane proteins that mediate the interaction between the cell and extracellular matrix ${ }^{27,28}$. Ligands such as COL11A1 and COMP are essential for the initial steps of the ECM-receptor interaction pathway. Integrins continue the pathway processes, culminating in different cell functions such as growth and regeneration ${ }^{29}$. Also, focal adhesion and PI3K-Akt signaling pathways specifically need the involvement of integrins to start their metabolic processes ${ }^{29}$.

The integrin gene ITGA10 was predicted to interact with COL11A1 and COMP. All analyses showing COL11A1, COMP, and ITGA10 also showed the TNMD gene. This gene possibly interacts with ITGA10 by the THBS4 gene, which is also part of the three connected pathways. Moreover, ITGA10 connects to TNC, involved in collagen formation. Thus, COL11A1, COMP, and TNMD take part in the three integrated pathways for $\mathrm{K}, \mathrm{P}, \mathrm{Na}$, $\mathrm{Mg}$, and $\mathrm{Cu}$ by its interaction with ITGA10. Their downregulation in $\mathrm{H}$-groups suggests that a high concentration of these minerals suppresses these pathways.

The ECM-receptor interaction pathway plays an essential role in skeletal muscle development ${ }^{30}$, which explains this pathway being found in muscle transcriptome. Simple diffusion of minerals can occur through pores in the tight junctions if the electrochemical gradient exists to push the ions through the pores ${ }^{31}$. The CD44 gene, a DEG for almost all minerals, has a possible role in tight junction regulation ${ }^{32}$. The relation of ECM-receptor interaction pathway to mineral concentration may be partially explained by the tight junctions' role in mineral absorption.

The protein digestion and absorption pathway was significant for six minerals ( $\mathrm{Ca}, \mathrm{Cu}, \mathrm{P}, \mathrm{Mg}$, Na, and $\mathrm{Se}$ ). The DEGs in this pathway encompass genes from the collagen family. Collagens are the most abundant protein in the ECM and take part in cell adhesion regulation, cell migration, and direct tissue development, the latest initiating after modifications in the ECM structure mediated by substrates ${ }^{6}$. These results indicate that ECM-interactions are related to mineral concentration regulation for most of the minerals in this study.

Zn and Cu antagonism on fatty acid metabolism. Fatty acid metabolism pathway (FAM) was enriched in $\mathrm{Zn}$ and $\mathrm{Cu}$ analyses. $\mathrm{Cu}$ analysis identified five DEGs in this pathway, ACACA, FASN, SCD, ELOVL6 and ELOVL5. From these, FASN, SCD and ELOVL6 were the only genes for Zn content in the same pathway. They all showed interactions between their encoded proteins. All DEGs included in this pathway were downregulated in $\mathrm{Cu}$ and upregulated in $\mathrm{Zn}$ analyses.

Animals with clinical $\mathrm{Cu}$ deficiency tend to accumulate fat due to disturbances in $\mathrm{FAM}^{33}$, and $\mathrm{Zn}$ has an antagonistic relationship in this phenomenon ${ }^{34}$. The five FAM related genes involved in Cu analysis take part in the cytoplasmic portion of the pathway, in which fatty acids biosynthesis occurs by the addition of one or more acetyl-CoA molecules, doubling the number of carbons in the fatty acid molecule produced in each cycle, as per KEGG data (https://www.genome.jp/kegg-bin/show_pathway?map01212).

Fatty acid biosynthesis can start with the co-enzyme Acetyl-CoA carboxylase, the product of ACACA, that catalyzes the carboxylation of acetyl-CoA to malonyl-CoA ${ }^{35}$. Subsequently, the product of FASN is responsible for the elongation of the fatty acid chains to precursors with 16 carbons. The elongation to 18 carbons requires the product of $E L O V L 6^{35}$. After that, the Stearoyl-CoA desaturase enzyme, which is the product of SCD, catalyzes the 
synthesis of Oleic acid $^{31}$. Cu is a cofactor of this enzyme $\mathrm{s}^{36}$ and, in the presence of this mineral, the FAM progresses just until the production of fatty acids with 20 carbons by the product of $E L O V L 5^{31}$, because it inhibits the production of Linoleic acid by increasing the Oleic acid synthesis ${ }^{36}$. The downregulation of ACACA, FASN, ELOVL6, $S C D$, and ELOVL5 in the $\mathrm{H}-\mathrm{Cu}$ group can explain the inhibition of long-chain fatty acids and fat accumulation under low $\mathrm{Cu}$.

A second hypothesis is that malonyl-CoA can also be the switch from fatty acids biosynthesis to fatty acids oxidation and energy production, which can lead to less fatty acid biosynthesis ${ }^{35}$. In rabbits, copper supplementation in the diet decreased the intramuscular fat content by improving fatty acid uptake and fatty acid oxidation ${ }^{37}$. This switch depends on the regulation of malonyl-CoA. For example, in ketosis, ketonic bodies accumulate in the tissue, and the activation of malonyl-CoA activates AMPK. This activation breaks malonyl-CoA, stopping the biosynthesis and starting the oxidation of fatty acids ${ }^{35}$. The AMPK signaling pathway was enriched for $\mathrm{Cu}$.

The second hypothesis can be reinforced by the simultaneous presence among DEGs for Cu of the genes FASN, ACACA and SCD, belonging both to AMPK and FAM pathways, as well as ADIPOQ, PCK 2 and LEP genes, which are exclusive from the AMPK pathway. Thus, animals with less $\mathrm{Cu}$ can have higher fat accumulation by biosynthesis (FAM) ${ }^{38}$ or oxidation (AMPK signaling pathway) ${ }^{37}$; probably by both processes.

$\mathrm{Zn}$ is a known $\mathrm{Cu}$ antagonist in FAM, due to its role in the stimulation of linoleic acid desaturation ${ }^{38}$. In the $\mathrm{Zn}$ analysis, we did not identify the ACACA gene as a DEG. Therefore, we hypothesized that, in this case, the product of FASN does the first step of fatty acid synthesis. As already discussed, the pathway continues to the precursor of oleic acid. However, in the presence of high $\mathrm{Zn}$, the pathway does not stop on fatty acids with 20 carbons ${ }^{29}$. Zn stimulates the linoleic acid desaturation and the production of long-chain fatty acids ${ }^{29}$.

In Japanese Black Cattle, there is a low negative correlation between $\mathrm{Cu}$ concentration and oleic acid $(-0.15)$, between $\mathrm{Cu}$ and linoleic acid $(-0,29)$, and between $\mathrm{Zn}$ and linoleic acid $(-0.05)^{39}$. This breed has more intramuscular fat than European cattle breeds. In our population, we did not identify a significant correlation between the GEBV s for oleic acid and the GEBV s for $\mathrm{Zn}$ and $\mathrm{Cu}$ concentration. We found a weak positive correlation $(\mathrm{r}=0.23)$ between linoleic acid and Cu GEBVs (data not shown). The absence of higher correlations can be attributed to the little variation of these minerals ${ }^{15}$ and fat deposition in our samples ${ }^{40}$. The samples used in the two contrasting groups for $\mathrm{Cu}$ and $\mathrm{Zn}$ analyses did not present significant $(\mathrm{p}>0.05)$ difference for seven fatty acids concentrations obtained elsewhere ${ }^{41}$ (data not shown). Also, our animals did not exhibit a clinical deficiency of these minerals. Thus, we can assume that, even if the difference in expression did not lead to a significant increase in fat, animals with low $\mathrm{Cu}$ concentration present modifications in FAM.

PPAR signaling pathway, enriched in $\mathrm{Cu}$ analysis, was also identified and is related to FAM. PPAR is one of the significant adipogenesis activators ${ }^{42}$. Only PLIN1 gene was in the other fatty acid associated pathways. This gene was found downregulated in $\mathrm{Cu}$, like all the other FAM related genes, and its product is involved directly in lipid metabolism ${ }^{43}$ and adipocyte differentiation ${ }^{44}$. LEP gene is also related to $\mathrm{Cu}$ and $\mathrm{Zn}$ and has an alleged role in the PPAR pathway regulation. It has a well-known relationship with obesity and stimulus for fatty acid oxidation ${ }^{45}$. As all the DEGs mentioned in FAM, LEP was upregulated in $\mathrm{Zn}$ and downregulated in $\mathrm{Cu}$ analyses and interacted with all DEG products in this pathway, when considering $\mathrm{Cu}$, and with FASN and $S C D$, when considering Zn. FAM genes were already shown to be related to iron concentration in a differential expression analysis with samples from the same population used in this study ${ }^{14}$.

We retrieved high similarity with known proteins for two non-annotated DEGs for $\mathrm{Cu}$. One of them, downregulated for $\mathrm{Cu}$, is similar to the mouse SENP3 gene. This gene has high similarity to other SENP family protein gene, SENP2 ${ }^{46}$. Both encode proteases that release SUMO3 and SUMO2 monomers, involved in several biological processes $^{46}$. Regarding fat deposition, overexpression of SENP2 increases fatty acid oxidation by upregulating the expression of enzymes linked to this process ${ }^{47}$. This non-annotated DEG can corroborate the hypothesis of the involvement of $\mathrm{Cu}$ concentration in fatty acid oxidation in cattle.

The THRSP gene, identified as upregulated for $\mathrm{Cu}$ and $\mathrm{Zn}$, encodes a nuclear protein involved in fatty acid synthesis ${ }^{48}$ interacting with FASN and ELOVL6. THRSP upregulation activates FASN ${ }^{49}$, being a candidate to the mechanism of FAM regulation by $\mathrm{Zn}$.

Among the other four genes downregulated for $\mathrm{Cu}, P C K 2$ is a candidate for obesity ${ }^{50}$ and is part of AMPK and PPAR signaling pathways. This gene's product interacts with ACACA. It has an impact in FAM by receptor interaction and changes in RBP4 gene, which plays a role in non-alcoholic fatty liver disease and can contribute to insulin resistance ${ }^{51}$. All these genes and pathways linking $\mathrm{Cu}$ and $\mathrm{Zn}$ to lipid metabolism can explain the genetic mechanisms underlying $\mathrm{Cu}$ associations to FAM and $\mathrm{Zn}$ antagonism in these processes.

Pathways enriched for just one mineral. COMP, FCGR $3 A, B L A-D Q B$, and THBS4 genes are involved in the phagosome pathway, all downregulated for K. $B L A-D Q B$ encodes an antigen, and the other genes encode glycoproteins with already known roles in phagocytosis. Potassium channels are known to modulate changes in the membrane during phagocytosis ${ }^{52}$, which can explain the relationship between the expression of these genes and $\mathrm{K}$ concentration.

The genes $C 1 Q B, C 1 Q C, C 7$, and NCAM1 were downregulated for Ca and partake in the prion disease pathway. The first two genes showed an interaction, and they encode proteins that form the complement component 1 , involved in the immune complement system. These genes are linked to the LAPTM5 gene, which encodes a lysosomal transmembrane protein. $C 7$ gene encodes a serum protein involved in the immune system and is connected to C1QTNF3, a gene that encodes another protein involved in the immune complement system. NCAM1 gene encodes a protein that is a cell adhesion linked to $C D 44$, part of the ECM-receptor pathway, showing that all pathways detected in this study are linked. 


\section{Conclusion}

By comparing the expression of genes in muscle samples with contrasting mineral concentrations, we hypothesized that the genetic regulation core for all minerals studied, except $\mathrm{Zn}$, resides in events of extracellular matrix interaction. ECM-receptor interaction, focal adhesion, and PI3k-Akt signaling pathways seem to be related to K, $\mathrm{P}, \mathrm{Na}, \mathrm{Mg}, \mathrm{Cu}$, and $\mathrm{Ca}$ content profiles in skeletal muscle. We also pointed out genes that may explain $\mathrm{Cu}$ and $\mathrm{Zn}$ association to adipogenesis-related pathways, as well as their antagonism on fat accumulation. Future studies can target our raised hypotheses and validate our DEGs to elucidate these biological mechanisms, since our main goal was in silico prediction.

\section{Methods}

Animals. All animal and experimental procedures were carried out following the guidelines provided by the Institutional Animal Care and Use Committee Guidelines of Embrapa Pecuária Sudeste ethics committee (São Carlos, São Paulo, Brazil. Protocol CEUA 01/2013). The Ethical Committee of Embrapa Pecuária Sudeste (São Carlos, São Paulo, Brazil) approved all experimental and animal protocols (approval code CEUA 01/2013). A group of 133 Nelore steers composes our samples that previous projects already used to produce data for mineral concentration $^{15}$, and RNA-Seq ${ }^{14}$. The entire sample group comes from a population of 373 Nelore steers fathered by 34 purebred Nelore sires in half-sibling families.

The animals used in our work result from artificial insemination, were born in two different breeding seasons, in two different farms. Approximately at 21 months of age, all animals used in this research were transferred and maintained in a feedlot at Embrapa Pecuária Sudeste (São Carlos, São Paulo, Brazil). After a 28 days adaptation period, they received food, water, and had a similar nutritional and sanitary management until the slaughter. The animals had ad libitum feed access twice a day with 5\% refusals, discarded daily. The diet contained $40 \%$ of dry matter constituted by corn silage, crude protein, ground corn, soybean meal, cottonseed, soybean hull, limestone, mineral mixture, urea and monensin $\left(\right.$ Rumensin $\left.^{\circledR}\right)$.

Mineral concentration genetic breeding value and contrasting groups. Mineral concentrations were measured as described elsewhere ${ }^{6}$ from Longissimus thoracis muscle steaks sampled between $11^{\text {th }}$ and $13^{\text {th }}$ ribs. Briefly, the samples were lyophilized and digested with microwave assistance using a closed-vessel microwave digestion system (Ethos-1600, Milestone-MLS, Sorisole, Italy). The mineral quantification was obtained in the Vista Pro-CCD ICP-OES spectrometer with a radial view (Varian, Mulgrave, Australia). Among measured minerals we selected Calcium (Ca), Copper ( $\mathrm{Cu}$ ), Potassium (K), Magnesium (Mg), Sodium (Na), Phosphorus (P), Sulfur (S), Selenium (Se), and Zinc (Zn) for our analyses because they have distinguished extreme animal groups.

The genetic breeding values (GEBV) for all mineral's concentration were estimated elsewhere ${ }^{15}$ for 373 animals encompassing our samples using a Bayesian model implemented in GenSel software ${ }^{53}$. The model considered contemporary groups formed by birthplace, feedlot location, and breeding season as fixed effects and age at slaughter as a covariate. The GEBVs were used to select 12 animals for each mineral with extreme phenotypes (six with high GEBV, called $\mathrm{H}$, and six with low GEBV, called L).

RNA-Seq data. We used muscle samples from all animals in each contrasting group for RNA extraction and RNA-Seq analysis as described elsewhere ${ }^{14}$. Total RNA was extracted using TRIzol ${ }^{\circledR}$ (Life Technologies, Carlsbad, CA). Its integrity was analyzed in a Bioanalyzer $2100^{\circledR}$ (Agilent, Santa Clara, CA, USA). Library preparation for RNA-Seq analysis was carried out using the TruSeq RNA Sample Preparation Kit (Illumina, San Diego, CA). Sequencing was carried out in an Illumina HiSeq $2500^{\circledR}$. All laboratory procedures were carried out in ESALQ Genomics Center (Piracicaba, SP, Brazil).

DEGs identification. RNA-Seq data obtained from muscle samples belonging to contrasting groups for a given mineral were used to determine DEGs for each mineral. The pipeline was as described in ${ }^{14}$ with the insertion of StringTie v1.2.2 $2^{54}$ instead of Cufflinks ${ }^{18}$ in Tuxedo Suite pipeline ${ }^{18}$.

SeqClean software (http://sourceforge.net/projects/seqclean/files/) was used to trim low-quality sequences and adapters. TopHat software v2.0.1 ${ }^{18}$ was used to align reads to the reference bovine genome (Bos taurus UMD 3.1, http://www.ensembl.org/Bos_taurus/Info/Index). After that, the StringTie v1.2.2 ${ }^{54}$ was used to assemble the transcripts and to estimate their expression levels, normalized as FPKM (fragments per kilobases per million). Cuffdiff v2.2.1 ${ }^{18}$ was then used to test for differential expression, calculating the average of each gene expression among the samples of the same contrasting group and calculating the FC. Only transcripts that passed the threshold of at least ten fragments aligned entered the differential expression test.

We performed a functional annotation analysis using Trinotate pipeline (http://trinotate.sourceforge.net/) to identify possible functions of non-annotated and predicted differentially expressed proteins for the minerals.

Relationship among minerals. We used a pairwise Pearson correlation analysis for the GEBVs of all minerals to quantify their dependency. Also, we performed a Pearson correlation analysis between GEBV and raw concentration measure for each mineral in order to convey the reliability of the GEBVs. A t-test was applied to verify if the mean GEBVs of the samples for all contrasting groups would also be statistically different for any other mineral.

Biological processes and pathways. We performed enrichment analysis using DAVID v6.8 software ${ }^{20}$ to discover biological processes in which the DEGs are acting. To access known protein-protein interaction regarding DEGs and pathways in which they may participate, we used STRING v10.5 software ${ }^{55}$. 


\section{References}

1. Duan, Q. et al. Genetic polymorphisms in bovine transferrin receptor 2 (TFR2) and solute carrier family 40 (iron-regulated transporter), member 1 (SLC40A1) genes and their association with beef iron content. Anim. Genet. 43, 115-122 (2011).

2. Geesink, G. H. \& Koohmaraie, M. Effect of Calpastatin on Degradation of Myofibrillar Proteins by $\mu$-Calpain Under Postmortem Conditions. J. Anim. Sci. 77, 2685-2692 (1999)

3. Suttle, N. 13. Iron. Mineral nutrition of livestock, https://doi.org/10.1079/9781845934729.0000 (2010).

4. Morris, C. A. et al. Effects of quantitative trait loci and the myostatin locus on trace and macro elements (minerals) in bovine liver, muscle and kidney. Anim. Genet. 44, 361-368 (2013).

5. Somogyi, T., Hollo, I., Csapo, J., Anton, I. \& Hollo, G. Mineral Content of Three Several Muscles From Six Cattle Genotypes. Acta Aliment. 44, 51-59 (2015).

6. Tizioto, P. C. et al. Calcium and potassium content in beef: Influences on tenderness and associations with molecular markers in Nellore cattle. Meat Sci. 96, 436-440 (2014).

7. Holló, G. et al. Effect of feeding on the composition of longissmus muscle of Hungarian Grey and Holstein Friesian bulls. Arch. Tierzucht 50, 575-586 (2007).

8. Mateescu, R. G. et al. Genetic parameters for concentrations of minerals in longissimus muscle and their associations with palatability traits in angus cattle. J. Anim. Sci. 91, 1067-1075 (2013).

9. Bonny, O. \& Bochud, M. Genetics of calcium homeostasis in humans: Continuum between monogenic diseases and continuous phenotypes. Nephrol. Dial. Transplant. 29, iv55-iv62 (2014).

10. Udensi, U. K. \& Tchounwou, P. B. Potassium Homeostasis, Oxidative Stress, and Human Disease. Int. J. Clin. Exp. Physiol. 4, 15-20 (2017).

11. Martens, H., Leonhard-Marek, S., Röntgen, M. \& Stumpff, F. Magnesium homeostasis in cattle: absorption and excretion. Nutr. Res. Rev. 25, 1-17 (2018).

12. Dietrich, N., Schneider, D. L. \& Kornfeld, K. A pathway for low zinc homeostasis that is conserved in animals and acts in parallel to the pathway for high zinc homeostasis. Nucleic Acids Res. 45, 11658-11672 (2017).

13. Schlecht, U. et al. A functional screen for copper homeostasis genes identifies a pharmacologically tractable cellular system. BMC Genomics 15, 1-14 (2014).

14. Da Silva Diniz, W. J. et al. Iron content affects lipogenic gene expression in the muscle of Nelore beef cattle. PLoS One 11, 1-19 (2016).

15. Tizioto, P. C. et al. Detection of quantitative trait loci for mineral content of Nelore longissimus dorsi muscle. Genet. Sel. Evol. 47, 1-9 (2015).

16. Buitenhuis, B., Poulsen, N. A., Larsen, L. B. \& Sehested, J. Estimation of genetic parameters and detection of quantitative trait loci for minerals in Danish Holstein and Danish Jersey milk. BMC Genet. 16, 1-8 (2015).

17. G. Hill, W. Quantitative Genetics in the Genomics Era. Curr. Genomics 13, 196-206 (2012).

18. Trapnell, C. et al. Transcript assembly and abundance estimation from RNA-Seq reveals thousands of new transcripts and switching among isoforms. Nat. Biotechnol. 28, 511-515 (2011).

19. Hu, S. et al. SAMHD1 Inhibits LINE-1 Retrotransposition by Promoting Stress Granule Formation. PLoS Genet. 11, 1-27 (2015).

20. Huang, D. W., Sherman, B. T. \& Lempicki, R. A. Systematic and integrative analysis of large gene lists using DAVID bioinformatics resources. Nat. Protoc. 4, 44-57 (2009).

21. Kanehisa, M., Furumichi, M., Tanabe, M., Sato, Y. \& Morishima, K. KEGG: New perspectives on genomes, pathways, diseases and drugs. Nucleic Acids Res. 45, D353-D361 (2017).

22. Gorlov, I. P., Gallick, G. E., Gorlova, O. Y., Amos, C. \& Logothetis, C. J. GWAS meets microarray: Are the results of genome-wide association studies and gene-expression profiling consistent? Prostate cancer as an example. PLoS One 4 (2009).

23. Jayawardena, D. P., Heinemann, I. U. \& Stillman, M. J. Biochemical and Biophysical Research Communications Zinc binds noncooperatively to human liver metallothionein 2a at physiological pH. Biochem. Biophys. Res. Commun. 493, 650-653 (2017).

24. Kayaalti, Z., Aliyev, V. \& Söylemezoğlu, T. The potential effect of metallothionein 2 A -5 A/G single nucleotide polymorphism on blood cadmium, lead, zinc and copper levels. Toxicol. Appl. Pharmacol. 256, 1-7 (2011).

25. Giacconi, R. et al. The $+838 \mathrm{C} / \mathrm{G}$ MT2A Polymorphism, Metals, and the Inflammatory/Immune Response in Carotid Artery Stenosis in Elderly People. Mol. Med. 13, 388-395 (2007).

26. Kohler, H. R., Rahman, B., Graff, S., Berkus, M. \& Triebskorn, R. Expression of the stree-70 protein family (hsp70) due to heavy metal contamination in the slug, deroceras reticulatum: and approach to monitor sublethal stress conditions. Chemosphere. 33, 1327-1340 (1996).

27. Faye Hui Chen, Ashby O., Thomas, Jacqueline T., Hecht, Mary B., Goldring, J. L. Cartilage Oligomeric Matrix Protein/ Thrombospodin 5 Supports Chondrocyte Attachment through Interaction with Integrins. J. Biol. Chem. 292, 342-351 (2005).

28. Li, Y. et al. A fibrillar collagen gene, Coll1a1, is essential for skeletal morphogenesis. Cell 80, 423-430 (1995).

29. Ivaska, J. Unanchoring integrins in focal adhesions. Nat. Cell Biol. 14, 981-983 (2012).

30. Thorsteinsdóttir, S., Deries, M., So, A. \& Bajanca, F. The extracellular matrix dimension of skeletal muscle development. Develp. Biol. 354, 191-207 (2011).

31. Goff, J. P. Invited review: Mineral absorption mechanisms, mineral interactions that affect acid-base and antioxidant status, and diet considerations to improve mineral status. J. Dairy Sci. 1-51, https://doi.org/10.3168/jds.2017-13112 (2018).

32. Kirschner, N. et al. CD44 regulates tight-junction assembly and barrier function. J. Invest. Dermatol. 131, 932-943 (2011).

33. Engle, T. E. Copper and lipid metabolism in beef cattle: A review. J. Anim. Sci. 89, 591-596 (2011).

34. Morris, C. A., Amyes, N. C. \& Hickey, S. M. Genetic variation in serum copper concentration in Angus cattle. Anim. Sci. 82, 798-803 (2006).

35. Foster, D. W. Malonyl-CoA: The regulator of fatty acid synthesis and oxidation. J. Clin. Invest. 122, 1958-1959 (2012).

36. Cunnane, S. C. Zinc and copper interact antagonistically in the regulation of linoleic acid metabolism. Prog. Lipid. Res. 20, 601-603 (1981).

37. Lei, L., Xiaoyi, S. \& Fuchang, L. Effect of dietary copper addition on lipid metabolism in rabbits. Food Nutr. Res. 61, 1348866 (2017).

38. Cunnane, S. C. Differential regulation of essential fatty acid metabolism to the prostaglandins: possible basis for the interaction of zinc and copper in biological systems. Prog. Lipid. Res. 21, 73-90 (1982).

39. Kitagawa, T., Funaba, M. \& Matsui, T. Relationships between mineral concentrations and physicochemical characteristics in the Longissimus thoracis muscle of Japanese Black cattle. Anim. Sci. J. 89, 211-218 (2018)

40. Cesar, A. S. Genome-wide association study for intramuscular fat deposition and composition in Nellore cattle. BMC Genom. 15, $1-15(2014)$.

41. Cesar, A. S. M. et al. Differences in the skeletal muscle transcriptome profile associated with extreme values of fatty acids content. BMC Genom. 17, 1-16 (2016)

42. Brun, R. P. et al. Differential activation of adipogenesis by multiple PPAR isoforms. Genes Dev. 10, 974-984 (1996).

43. Tansey, J. T., Sztalryd, C., Hlavin, E. M., Kimmel, A. R. \& Londos, C. The central role of perilipin A in lipid metabolism and adipocyte lipolysis. IUBMB Life 56, 379-385 (2004).

44. Lyu, Y. et al. Defective differentiation of adipose precursor cells from lipodystrophic mice lacking perilipin 1. PLoS One 10, 1-18 (2015). 
45. Minokoshi, Y., Okamoto, S. \& Toda, C. Regulatory role of leptin in glucose and lipid metabolism in skeletal muscle. Indian J. Endocrinol. Metab. 16, 562 (2012).

46. Tatham, M. H. et al. Polymeric Chains of SUMO-2 and SUMO-3 are Conjugated to Protein Substrates by SAE1/SAE2 and Ubc9. J. Biol. Chem. 276, 35368-35374 (2001).

47. Koo, Y. D. et al. SUMO-Specific Protease 2 (SENP2) is an important regulator of fatty acid metabolism in skeletal muscle. Diabetes 64, 2420-2431 (2015).

48. Donnelly., N. I. H. Public Access. 61, 114-122 (2009).

49. Yao, D. W. et al. Thyroid hormone responsive (THRSP) promotes the synthesis of medium-chain fatty acids in goat mammary epithelial cells. J. Dairy Sci. 99, 3124-3133 (2016).

50. Beale, E. G., Harvey, B. J. \& Forest, C. PCK1 and PCK2 as candidate diabetes and obesity genes. Cell Biochem. Biophys. 48, 89-95 (2007).

51. Romeo, S. \& Valenti, L. Regulation of retinol-binding protein 4 and retinol metabolism in fatty liver disease. Hepatology 64, $1414-1416(2016)$.

52. Demaurex, N. Functions of proton channels in phagocytes. Wiley Interdiscip. Rev. Membr. Transp. Signal. 1, 3-15 (2012).

53. Fernando, R. L. \& Garrick, D. J. GenSel-User manual for a portfolio of genomic selection related analyses. Anim. Breed. Genet. Iowa State Univ. Ames 0-24 (2008).

54. Pertea, M. et al. StringTie enables improved reconstruction of a transcriptome from RNA-seq reads. Nat. Biotechnol. 33, 290-295 (2015).

55. Szklarczyk, D. et al. The STRING database in 2017: Quality-controlled protein-protein association networks, made broadly accessible. Nucleic Acids Res. 45, D362-D368 (2017).

\section{Acknowledgements}

We thank FAPESP (2012/23638-8) for financing the projects encompassing this one and Capes for the scholarship for the first author. We thank all the Staff of Embrapa Pecuária Sudeste responsible for monitoring and taking care of animals and the research group of Dr. Ana Rita for collecting the mineral phenotypes. LCAR, GBM, ARAN and LLC are recipients of a CNPq research productivity scholarship.

\section{Author Contributions}

J.A., L.L.C., A.R.A.N., G.B.M. and L.C.A.R. designed the experiments and analysis. J.A., W.J.S.D., A.O.L., M.I.P.R., B.G.N.A., O.P., J.V.S., L.A.L. and C.F.G. performed the experiments and analysis. J.A., P.C.T., W.J.S.D., C.E.B., B.G.N.A., O.P. and M.R.S.F. interpreted the results. J.A. and L.C.A.R. drafted the manuscripts. All authors revised the manuscripts and read and approved the final manuscript.

\section{Additional Information}

Supplementary information accompanies this paper at https://doi.org/10.1038/s41598-019-49089-x.

Competing Interests: Author Polyana C. Tizioto was employed by company NGS Genomic Solutions. All the other authors declare no competing interests.

Publisher's note: Springer Nature remains neutral with regard to jurisdictional claims in published maps and institutional affiliations.

(c) (i) Open Access This article is licensed under a Creative Commons Attribution 4.0 International License, which permits use, sharing, adaptation, distribution and reproduction in any medium or format, as long as you give appropriate credit to the original author(s) and the source, provide a link to the Creative Commons license, and indicate if changes were made. The images or other third party material in this article are included in the article's Creative Commons license, unless indicated otherwise in a credit line to the material. If material is not included in the article's Creative Commons license and your intended use is not permitted by statutory regulation or exceeds the permitted use, you will need to obtain permission directly from the copyright holder. To view a copy of this license, visit http://creativecommons.org/licenses/by/4.0/.

(C) The Author(s) 2019 